terne, il apparaît sur les jeunes des taches blanches plus ou moins étendues.

\section{Productivité du type commun.}

10 Production laitière : Les aptitudes laitières de la chèvre de Maradi sont assez marquées, au point que dans de nombreux villages dépourvus de gros bétail elle est seule à fournir le lait.

La lactation dure six mois en moyenne. La production variable s'établit aux environs de 01.400 en saison sèche et 01.600 à 01.800 pendant l'hivernage.

La chèvre rousse est surtout d'une remarquable prolificité, les portées doubles sont de règle, mais les portées triples et même quadruples s'observent fréquemment.

20 Production de viande : La race de Maradi est également un animal de boucherie intéressant. De squelette léger, bien en muscle, les animaux fournissent une viande de bonne qualité, très savoureuse, qui constitue la base de l'alimentation carnee de l'indigène. La chair, beaucoup moins sèche que ne l'est ordinairement la viande de chèvre, possède une saveur agréable, certainement liée à la qualité des pâturages, car elle est caractéristique de la région de Maradi. Dans les zones d'élevage excentriques, les qualités boucherie sont, en effet, beaucoup moins bonnes. Les troupeaux vivants en région sèche fournissent une viande ordinaire, de faible rendement.

Dans l'ensemble, les rendements moyens varient de 45 à $50 \%$. Les jeunes mâles castrés, qui manifestent une remarquable aptitude à l'engraissement, dépassent largement ces moyennes et atteignent des rendements de 54 à $55 \%$.

La race de Maradi compte 600.000 têtes environ, en territoire français.

3o Production de peaux : C'est surtout comme productrice de peaux qu'est appréciée la race de Maradi. La vogue des peaux dites de "Sokoto" sur les marchés mondiaux n'est pas seulement liée à une question de mode, mais résulte de leur contexture fine et serrée qui les fait rechercher par l'industrie de la maroquinerie et de la chaussure de luxe.

L'artisanat local n'assure qu'un débouché négligeable à la production qui donne lieu à un important commerce d'exportation vers le Nigeria qui achète plus de 100.000 peaux par an.

\section{Production du type amélioré.}

Des essais d'amélioration par sélection sont en cours dans différents établissements appartenant aux. Sociétés de prévoyance locale. Ils sont placés sous le contrôle du Service de l'Élevage. Ces essais ont surtout pour but d'étendre l'aire d'extension de la race en vue de l'accroissement de la production peaussière. L'acclimatement dans les régions nouvelles est souvent difficile et les résultats ne sont pas toujours heureux.

L'amélioration ne porte ni sur le développement de la qualité laitière, ni sur celui des qualités boucherie. Les rendements indiqués ci-dessus ne sont donc pas modifiés.

\title{
L'élevage en Guyane française
}

\section{A. - ÉTAT ICTUEL DE LELEVAGE 10 Effectifs}

EN 1940, la situation du cheptel guyanais était la suivante :

\begin{tabular}{|c|c|c|}
\hline Chevaux et mulets & 175 têtes & en \\
\hline Anes $\ldots \ldots \ldots \ldots \ldots$ & 200 & - \\
\hline Bovins.............. & 3.500 & - \\
\hline Bubalins ........... & 600 & 一 \\
\hline Porcins..... & 7. 500 & 一 \\
\hline Caprins et ovins & 400 & - \\
\hline
\end{tabular}

Le cheptel pénitentiaire figure dans ces chiffres pour :

Chevaux ..................... 4

Bovins .................... 390

Bubalins ................... 380

Porcins....................... 365
Les chiffres ci-dessus montrent le peu d'importance du cheptel guyanais, surtout si on les compare aux chiffres de la population et de la superficie des terres utilisables. La population totale de la Guyane est de 47.000 habitants environ et la superficie des savanes, propres à l'élevage, de 300.000 hectares. Il y a donc approximativement l boeuf ou buffle sur 73 hectares et moins de 9 têtes de gros bétail pour 100 habitants.

L'èlevage guyanais fut autrefois (fin du XVII ${ }^{e}$ siècle) beaucoup plus florissant et la Guyane, réduite aujourd 'hui à importer du bétail de boucherie des pays voisins, fut exportatrice vers les Antilles. Mais sans remonter à cette époque lointaine, on recensait encore, en 1875, 16.000 bovins entre les rivières de Kourou et de Sinnamary et 12.000 sur les ternitoires d'Iracoubo et d'Organabo. 
Cette régression a eu des causes multiples dont les principales sont :

10 Un décret du 16 pluviose an II, taxant la viande à 8 sous par kilo, au moment mêmé où le Brésil et le Venezuela intensifiaient leur concurrence.

$2^{\circ} \mathrm{La}$ suppression de l'esclavage en 1704 et en 1848, qui a eu des répercussions fâcheuses sur l'élevage.

$3^{\circ}$ La découverte de l'or, 'qui provoqua la ruée vers l'intérieur du pays et par suite I'abandon des cultures et de l'élevage.

$4^{\circ}$ Les maladies épizootiques insuffisamment combattues.

\section{$2^{\circ}$ Zones propices à l'élevage}

Les zones propices à l'élevage sont situées sur le territoire de la Guyane française proprement dit. Le territoire de l'Inini, presque entièrement recouvert de forêts, ne saurait convenir à l'élevage, sauf en quelques points de faible importance.

Les savanes guyanaises sont situées le long de la côte entre Cayenne et la pointe des Hattes. Elles s'étendent de part et d'autre de la route coloniale $n^{\circ}$ 1, qui réunit Cayenne à Saint-Laurent-du-Maroni en suivant la côte. Elles sont séparées de l'Océan par une barrière presque ininterrompue de palétuviers qui ont poussé sur les prairies basses noyées.

Le climat de cette région est doux et régulier. Les temperatures extremes sont de $34^{\circ}$ pour le mois le plus chaud (septembre) et de $21^{\circ}$ pour. le mois le plus frais (janvier). La moyenne des températures annuelles oscille entre 25 et $27^{\circ}$, la saison des pluies est très longue, durant de la mi-novembre au début de juillet, avec, d'une part, un maximum en avril, mai, juin et, d'autre part, une interruption en février-mars. Il y a lieu de noter également que pendant la période la plus chaude (mi-août à miseptembre), de violents orages sont fréquents.

Les savanes ne constituent pas une zone absolument uniforme; elles sont, en effet, séparées les unes des autres par des rivières ou des parties boisées. Chaque savane s'étend sur une longueur de 10 à 20 kilomètres et sur une largeur presque égale et, comme nous l'avons signalé précédemment, leur ensemble couvre une surface totale de près de 300.000 hectares. Certaines savanes ne sont utilisables qu'en saison sèche, et d'autres en saison des pluies. Les premières sont, en effet, inondées pendant une partie de l'année et les pâturages des secondes perdent toute valeur sous l'influence de la sécheresse.

D'une façon générale; dans la zone des savanes, les rivières sont nombreuses et, de plus, le plan d'eau est peu profond et l'installation de puits $y$ serait facile.

Si nous examinons la composition botanique des pâturages, nous y trouvons surtout des graminées dont plusieurs constituent d'excellents fourrages. Il en est ainsi notamment pour les suivantes :

Sporobolus Indicus - Tapia grass. Assez commune en Guyane, peut donner un très bon foin quand la récolte est faite dans de bonnes conditions.

Cynodion dactylon - Bermuda grass - Chiendent. Croît sur n'importe quel sol. Ne craint pas. la sécheresse et est bien acceptee par les bovidés.

Axonopus compressus - Savannah grass Chiendent. Pousse bien surtout sur. le territoire guyanais. Considérée aux États-Unis, au Mexique, en diverses régions chaudes, comme l'une des meilleures graminées pour pâturages permanents. Persiste pendant la saison sèche'.

Panicum molle - Para grass - herbe de Para. Assez répandue en Guyane. Peut faire d'excellentes prairies dans les sols bas et humides.

Panicum maximum - Herbe de Guinée. Mélangée à d'autres graminées constitue un excellent fourrage.

Herbe à canne (nom créole) - Cạnarana (nom brésilien). Excellente graminée des savanes noyées de l'Oyapock, existerait également dans la région de la rivière de Kaw.

Sur le territoire de l'Inini, il existe deux centres d'élevage de faible importance : celui du HautMaroni et celui du Haut-Approuagne. Dans ce dernier centre, le cheptel comprendrait 400 à 500 bovins, plusieurs dizaines de chèvres, quelques moutons et quelques buffles. Certains placers du Haut-Maroni, le T.H.R. par exemple, par suite de l'existence de savanes, pourraient avoir un élevage plus important.

\section{B. - CARACTÈRES ACTUELS DE L'ÉLEVAGE Bovins et Bubalins}

Le cheptel bovin actuel résulte de la fusion des nombreüses races importées à différentes reprises depuis le XVIIe siecle. Il s'est ainsi formé une race spéciale, d'un format moyen, dite "race créole 》. Certains bœufs pèsent 350 à.400 kilos et donnent à la boucherie un rendement de $48 \%$. La vache en période de lactation donne 4 litres de lait par jour.

L'élevage est' pratiqué d'une façon très rúdimentaire. Dans les savanes, les animaux vivent en complète liberté, sans aucune surveillance et sans aucun soin, entièrement abandonnés à eux-mêmes.

Dans l'île de Cayenrne, quelques créoles, mais surtout des forçats libérés, en majorité des arabes, se livrent à l'élevage, possédant chacun 3 à 10 têtes de bétail. Leur but est la production du lait. Contrairement à ce qui se passe pour l'élevage dans les savanes, les animaux sont rentrés le soir. Mais quelle que, soịt la saison, ils ne reçoivent aucun complément de ration. Or, dạns I'île de Cayenne, 
les pâturages sont de dimensions réduites et à la période de sécheresse, les animaux n'y trouvent plus qu'une nourriture très insuffisante.

Les buffles sont utilisés pour les transports; leur élevage se pratique autour des villes. Ces animaux sont parqués le soir dans des enclos mal tenus et vont, le jour, pâturer dans les savanes voisines. Les 600 buffles existant actuellement proviennent des six importés en 1876. Cet accroissement montre que le buffle a trouvé en Guyane un habitat favorable.

\section{Porcins}

Le porc guyanais est mal conformé pour la production de la viande, il appartient au type coureur. Cette dégénérescence résulte des conditions de vie qui lui sont imposées. Si pendant la saison des pluies il trouve facilement sa nourriture, grâce aux fruits qui sont nombreux, en saison sèche il n'y a plus zien à manger. Durant ces dernières années, il semble que les planteurs de bananes aient entrepris un élevage plus rationnel. Par ailleurs, des crédits ont ćté mis à la disposition du service de la colonisation pour entreprendre, en administration directe, un élevage de porcs sur une bananeraie restée inculte et cédée à l'administration. Cette entreprise doit servir d'exemple et contribuer à réduire les importations de viande.

\section{Orins et Caprins}

Le climat humide de la Guyane ne paraît guère convenir à l'élevage du mouton. Les quelques individus qui existent sont groupés dans les régions sèches de l'île de Cayenne et appartiennent à la race Black-Head qui paraît s'y être acc'imatée.

Les chèvres sont groupées en petits troupeaux aux environs des villes. Elles sont généralement en bon état et rien ne paraît s'opposer à leur élevage.

\section{Chevaux}

La région de Sinnamary possède quelques chevaux qui vivent à l'état sauvage. On en rencontre quelques autres dans les villes, où ils sont utilisés pour les transports. Ces animaux sont de petite taille, mal conformés et le plus souvent en très mauvais état.

\section{Volailles}

L'élevage des volailles est assez répandu, surtout aux environs des villes. Un élevage de canards, qui paraît très bien réussir, a été entrepris par le service de la colonisation sur le pénitencier indochinois de Crique Anguille. Les produits, vendus aux enchères, constituent un apport non négligeable de viande pour le marché de Cayenne.

\section{Sociétés d'élevage}

Il n'existe pas de société d'élevage. La Compagnie d'Élevage de la Guyane française ne s'occupe que de l'importation des animaux de boucherie. Par contre, il existe quelques habitants se consacrant plus particulièrement à l'élevage : MM. Vernet, à Sinnamary, Raffray, à Iracoubo, et surtout Said, à Massa (270 bovins). Ces entreprises nous montrent qu'il est possible d'obtenir des résultats intéressants avec de faibles moyens financiers.

Le cheptel guyanais étant insuffisant, la colonie doit avoir recours aux importations pour assurer son ravitaillement en viandes et en lait ou produits dérivés.

\section{Viandes}

L'absence de frigorifique oblige la Guyane à importer du bétail vivant afin de pouvoir se procurer de la viande fraîche.

Pour la période 1922-1930, la moyenné annuelle de ces importations a été de 3.505 bovins. En 1933 , 1934 et 1937 elles ont été les suivantes :

$\begin{array}{lrrrr} & & 1933 & 1934 & 1937 \\ \text { Bovins } \ldots \ldots \ldots \ldots \ldots & 4.075 & 5.445 & 3.777 \\ \text { Porcs ........... } & 32 & 2 & 1 \\ \text { Moutons ......... } & - & - & 147 \\ \text { Chèvres.......... } & - & - & 96\end{array}$

Les deux principaux pays fournisseurs sont le Venezuela et le Brésil. L'importation du Venezuela se falt par un vapeur et celle du Brésil par des voiliers. Le transport du bétail est effectué dans de très mauvaises conditions. Le voyage eșt d'assez longue durée, cinq jours du Venezuela à Cayenne et trois à huit jours du Brésil à Cayenne. Les animaux sont entassés sur les ponts des bateaux et manquent souvent de fourrage et d'eau pendant le voyage. Certains meurent en route et d'autres arrivent très fatigués, en mauvais état et ne peuvent pas être livrés à la consommation.

La Guyane importe également des viandies salées et des conserves.

\section{Situation sanitaire}

Par suite de l'inexistence d'un véritable service vétérinaire, la pathologie animale de la Guyane n'est pas encore entièrement connue. Il n'est pas doûteux que les maladies ont joué un rôle important dans l'amoindrissement des effectifs, car les modestes recherches entreprises ont déjà permis d'identifier le charbon bactéridien, la trypanosomiase et la piroplasmose.

La prophylaxie anticharbonneuse donne lieu tous les ans à de nombreuses vaccinations : 1.735 en 1940 et 2.295 en 1941. Cette vaccination annuelle est 